\title{
The Manual Polybrene Method Can Be Used for the Pretransfusion Test to Mitigate Daratumumab Interference without a Supplementary Antiglobulin Test
}

\author{
Lin-Nan Shao Ni Wang Wei-Jian Yu Shi-Hang Zhou \\ Blood Group Reference Laboratory, Dalian Blood Center, Dalian, China
}

\section{Dear Editor,}

Daratumumab is an anti-CD38 monoclonal antibody approved in China by the National Medical Products Administration in July 2019 for the treatment of relapsed/ refractory multiple myeloma (MM). Once the use of daratumumab becomes widespread for the management of MM patients, it is possible that a higher number of blood samples will be submitted from such patients to transfusion laboratories. This will present a great challenge for transfusion services. This is due to the pan-agglutination of indirect antiglobulin test (IAT) caused by daratumumab which may be weakly positive in all media (e.g., saline, low ionic strength saline, and polyethylene glycol) with all IAT methods (e.g., gel, tube, or solid-phase) [1]. Currently, treating red blood cells (RBCs) with dithiothreitol (DTT) is the most-used method to mitigate interference [2]. However, the drawback of this method is that it is time-consuming, and CD38 antigen, along with other clinically significant RBC antigens like the Kell system, are denatured by it [3].

Polybrene is a stable aliphatic synthetic polymer with a positive charge. There is a possibility that it can induce reversible, nonspecific $\mathrm{RBC}$ agglutination and cause the approximation of RBCs and, as a result, permit the crosslinking formation by antibody molecules [4]. The manual polybrene test (MPT) was developed in the 1980s. Lack of adequate sensitivity for the Kell system is a major disadvantage of this method. The MPT was therefore considered unsuitable as a primary technique for extensive use and replaced by other methods in Western populations. However, the frequency of $\mathrm{K}$-positive individuals in oriental populations is close to zero. The MPT is thus used widely in southeast Asia.

To the best of our knowledge, only 1 previously published article has reported the use of the MPT to overcome the interference of daratumumab [5]. The method described in this particular article has not been confirmed by other transfusion laboratories.

We performed cross-matching and antibody screening tests with the MPT kit (Baso Diagnostics Inc. Zhuhai, China). The routine approach: 1 drop of 3-5\% RBCs were mixed with 2 drops of the test sera, and then added to 0.65 $\mathrm{mL}$ low ionic medium and 2 drops of polybrene solution. Centrifuged, the cell-free supernatant fluid was decanted, and 2 drops of resuspending solution were added. This was conducted in parallel with positive and negative controls of $2 \mathrm{MM}$ patients undergoing daratumumab therapy. With both the naked eye and microscopy, we observed no interference. Based on these observations, we hypothesized that the MPT lacks sensitivity for daratumumab. The abovementioned article by Yeh et al. [5] also suggested that a supplementary antiglobulin test could be added to compensate for the inadequate sensitivity in the Kell system. The results of our experiment were not in agreement with these findings. This is because there is a reappearance of pan-agglutination when the completion of the MPT is followed by washing the RBCs with saline which is then followed by a supplementary antiglobulin test.

The advantages of the MPT are that it is simple to perform, inexpensive, rapid (3-5 min), and does not destroy the

Lin-Nan Shao and Ni Wang contributed equally to this work. 
surface antigen of RBCs. We thus suggest that it can be used for the pretransfusion test to mitigate daratumumab interference without a supplementary antiglobulin test. Moreover, K-negative units should be provided while using the MPT method unless the patient is known to be K-positive.

\section{Statement of Ethics}

Informed consent was obtained from two participants. This study was approved by the Dalian Blood Center Ethics Committee.

\section{Conflict of Interest Statement}

The authors declare that they have no conflicts of interest relevant to this article.

\section{Author Contributions}

Original draft: Lin-Nan Shao; Investigation: Lin-Nan Shao, Ni Wang; Review and editing: Wei-Jian Yu, Shi-Hang Zhou.

\section{References}

1 AABB. [Internet] Association Bulletin \#1602. Mitigating the anti-CD38 interference with serologic testing [cited 2016]. Available from: http://www.aabb.org/programs/publications/bulletins/Documents/ab16-02.pdf

2 Lancman G, Arinsburg S, Jhang J, Cho HJ, Jagannath S, Madduri D, et al. Blood Transfusion management for patients treated with anti-CD38 monoclonal antibodies. Front Immunol. 2018 Nov;9:2616.
3 Bub CB. Dithiothreitol treatment of red blood cells. Immunohematology. 2017 Dec;33(4): 170-2.

4 Lalezari P, Jiang AF. The manual polybrene test: a simple and rapid procedure for detection of red cell antibodies. Transfusion. 1980 Mar-Apr;20(2):206-11.

5 Yeh TJ, Yeh CJ, Liu YC, Hsiao HH. Manual polybrene method for pretransfusion test could overcome the interference of daratumumab therapy in myeloma. Transfusion. 2019 Aug;59(8):2751-2. 